\title{
A rare case of severe pulmonary hypertension in pregnancy
}

\author{
Ramna Banerjee*, Farheen Rahman
}

Department of Obstetrics and Gynaecology, Apollo Gleneagles Hospital, Kolkata, West Bengal, India

Received: 13 October 2020

Revised: 19 October 2020

Accepted: 21 October 2020

\section{*Correspondence:}

Dr. Ramna Banerjee,

E-mail: rban44@rediffmail.com

Copyright: ( ) the author(s), publisher and licensee Medip Academy. This is an open-access article distributed under the terms of the Creative Commons Attribution Non-Commercial License, which permits unrestricted non-commercial use, distribution, and reproduction in any medium, provided the original work is properly cited.

\begin{abstract}
Heart diseases in pregnancy have always been a source of major concern for both the patients as well as the caregivers because of the high risk of maternal morbidity and mortality and the need for specialist care by a multidisciplinary team preferably in a tertiary care hospital. Amongst the whole gamut of heart diseases, pulmonary hypertension is associated with high maternal morbidity and mortality (in the order of $20 \%$ in some studies) and hence is considered a contraindication for pregnancy. We report a rare case of a lady at 36 weeks gestation in her second pregnancy, with a previous caesarean section, who was incidentally diagnosed with severe pulmonary hypertension (PAP-110 mmHg) during her antenatal check-up in the third trimester of pregnancy and survived without any major complications after being promptly referred to and treated in a multispecialty hospital. Her previous pregnancy was absolutely uneventful and even in this pregnancy she had very mild symptoms as compared to the severity of the disease found on further investigation.
\end{abstract}

Keywords: Primary pulmonary hypertension, Echocardiography, Multidisciplinary

\section{INTRODUCTION}

Heart diseases in pregnancy have always been a source of major concern for both the patients as well as the caregivers because of the high risk of maternal morbidity and mortality and the need for specialist care by a multidisciplinary team preferably in a tertiary care hospital.

Nowadays, most heart diseases, especially congenital ones, are identified early on and treated before the woman becomes pregnant. However, especially in a developing country like India, many women are diagnosed for the first time when they come for their routine antenatal checkups or when they present with symptoms suggestive of heart disease whilst already being pregnant.

Amongst the whole gamut of heart diseases, pulmonary hypertension is associated with high maternal morbidity and mortality (in the order of $20 \%$ in some studies) and hence is considered a contraindication for pregnancy. ${ }^{1,2}$

Pulmonary hypertension is defined as increase in mean pulmonary arterial pressure (MAP) of $25 \mathrm{mmHg}$ or more at rest as assessed by right heart catheterisation. It is a rare condition whose cause may be idiopathic or due to underlying conditions like congenital heart diseases, lung diseases, chronic thromboembolism of pulmonary vasculature, connective tissue disorders which lead to gradually progressive narrowing of the pulmonary blood vessels and endothelial dysfunction eventually leading to right sided heart failure and death. ${ }^{3}$

In pregnancy it usually presents with progressive exertional dyspnoea and fatigue. This is due to reduced cardiac output and impaired oxygen transport. Since these symptoms also occur in healthy pregnant women, the diagnosis during pregnancy can be delayed. In severe cases patients may present with chest pain and syncopal 
attacks, as the normal physiological changes of pregnancy are poorly tolerated by such patients. ${ }^{4}$

Any woman with this condition planning to get pregnant should be properly counselled about the high risk involved and ideally should be advised to avoid pregnancy or undergo early termination even though termination is also associated with maternal risks.

We report a rare case of a lady at 36 weeks gestation in her second pregnancy, with a previous caesarean section, who was incidentally diagnosed with severe pulmonary hypertension (PAP-110 mmHg) during her antenatal checkup in the third trimester of pregnancy and survived without any major complications after being promptly referred to and treated in a multispecialty hospital. Her previous pregnancy was absolutely uneventful and even in this pregnancy she had very mild symptoms as compared to the severity of the disease found on further investigation.

\section{CASE REPORT}

A 37 year old woman, second gravida $(\mathrm{P} 1+0)$ booked at 36 weeks gestation in our hospital. She had delivered by casarean section 8 years back. She had conceived spontaneously this pregnancy. She was originally booked at a district general hospital and had been referred to a tertiary unit for further management and delivery in view of incidental finding of moderate pulmonary hypertension on echocardiogram. The echocardiogram was done at 34 weeks gestation due to complain of occasional shortness of breath for 2 weeks. She did not give any history of syncope or palpitation or chest pain. She had a echo done that showed left ventricle was normal in size, shape and wall thickness with adequate systolic and diastolic function. Left atrium was normal. Right atrium and right ventricle were borderline enlarged. All valves appeared morphologically normal except moderate tricuspid regurgitation. Estimated pulmonary arterial pressure was $70 \mathrm{mmHg}$ (systolic).

Her observations in OPD in hospital showed BP of $100 / 70$; weight of $58 \mathrm{~kg}$; pulse of $80 / \mathrm{m}$, respiratory rate of $16 / \mathrm{min}$. Abdominal examination showed symphysis fundal height was $33 \mathrm{~cm}$, uterus was soft and relaxed and there was no evidence of scar tenderness on palpation. Her first trimester combined screening test and anomaly scan in the current pregnancy were normal. All her booking bloods were normal. She had had an obstetric ultrasound at 32 weeks that showed normal growth and liquor volume and Doppler's.

She was referred to the anaesthetist and cardiologist in view of her findings and history. An obstetric ultrasound was arranged along with a complete blood count, random blood sugar, thyroid profile, urea, creatinine and electrolytes. She had an echocardiogram done at hospital that showed normal left ventricle and left atrium. The right atrium was enlarged with eccentric hypertrophy of the right ventricle $(2.69 \mathrm{~cm})$. The mitral and aortic valves appeared normal with no significant regurgitation. There was no evidence of left to right shunt with negative contrast injection. Tricuspid valve showed normal leaflets, dilated annulus and grade 3+ regurgitation. Pulmonic valve showed normal cusps and good systolic function. Main pulmonary artery was dilated. Ventricular and intrarterial septum was intact. Pericardium appeared normal and there was no effusion. Estimated pulmonary artery pressure was $110 \mathrm{mmHg}$ (systolic).

She had an obstetric USG done that showed the fetus appeared small for date with growth tailing off to less than $50^{\text {th }}$ centile and increased end diastolic flow in the middle cerebral artery suggestive of early redistribution. In view of these findings and history of less fetal movements patient was administered steroids and delivery was planned. She was reviewed by the cardiologist and anaesthetist and a high risk of right heart failure post delivery was discussed and documented. She was also reviewed by the paediatrician and need for monitoring the baby in special care baby unit was discussed in view of deranged Doppler's.

Caesarean section was done under combined spinal epidural anaesthesia. Continuous invasive arterial blood pressure monitoring was instituted prior to induction of anesthesia. One large-bore peripheral line was placed, and a multilumen (right internal jugular vein, triple lumen) central venous catheter was done for continuous monitoring of central venous pressure (CVP) and the eventual application of vasopressors/inotropes if needed. Standard ECG monitors, pulse oximeter was used and intake/output was strictly monitored.

She was shifted to intensive care unit after delivery. Post operative period was uneventful. She needed oxygen support at 2 litres per minute for the first 2 days at which her oxygen saturation remained at $96 \%$. Blood pressure and pulse rate remained stable throughout (130/80; $82 / \mathrm{min}$ ). Postoperative analgesia was managed with intramuscular tramadol and fentanyl via PCA pump. She was monitored very carefully for any signs of heart failure. She received clexane for thromboprophylaxis along with TEDS stockings. Oxygen was gradually weaned off and she was shifted to the ward on $3^{\text {rd }}$ postoperative day. She was started on bosentas $(62.5 \mathrm{mg})$ twice daily and toresamide $5 \mathrm{mg}$ from fourth postoperative day by the cardiologist. In view of these medicines breast feeding was suppressed with cabergoline.

A ventilation/perfusion scan and duplex ultrasound (US) of the lower limbs excluded pulmonary embolic disease. Blood work up for vasculitis screen did not reveal any evidence of vasculitis or connective tissue disease. She and baby were discharged home on the eight postoperative day. 


\section{DISCUSSION}

Where primary pulmonary hypertension is not diagnosed until late pregnancy an elective delivery with caesarean section is preferred. ${ }^{5}$ This facilitates a multidisciplinary management of the patient to optimise outcomes and prevents added load on the heart that can lead to cardiorespiratory failure. In current case report we have presented a 37 year old woman who was incidentally diagnosed with severe pulmonary hypertension on being investigated for occasional shortness of breath in late pregnancy. She had no problems pre-pregnancy and did not require any medication for pulmonary hypertension during pregnancy and delivered a healthy baby near term.

Pulmonary hypertension is poorly tolerated during pregnancy. Deterioration typically occurs in the second trimester with symptoms of fatigue, dyspnoea, syncope and chest pain. This corresponds to the physiological increase in cardiac output and blood volume of $40 \%$.

Since maternal cardiac output and blood volume can increase by 30 to $50 \%$ during the third trimester pregnancy is strongly discouraged in patients with pulmonary arterial hypertension and, if pregnancy occurs, early termination due to stress and pain of labour.

Regional anaesthesia is generally preferred over general anaesthesia because regional anaesthesia has less effect on systemic vascular tone and cardiac function. Maternal mortality may increase with general anaesthesia. Approximately 70 to $75 \%$ of the patients are delivered under epidural anaesthesia. ${ }^{6}$

Patients with primary pulmonary hypertension are at risk of thromboembolism, hence thromboprophylaxis is very essential. Vasodilators may be needed but in our case since the patient was stable with minimal symptoms, they were not needed prior to delivery. Attention should be given to use of appropriate antibiotics to prevent infection and adequate oxygenation and fluid maintenance to prevent heart failure.

\section{CONCLUSION}

Pulmonary hypertension is a rare heart disease with high mortality and morbidity for the mother. It is one of the conditions in which pregnancy is contraindicated but if the woman still chooses to continue with pregnancy then she needs to be managed in a highly specialised multidisciplinary centre with a good intensive care set up. Many times the symptoms are masked as the normal symptoms of pregnancy, leading to a delay in diagnosis and appropriate intervention.

So only a high degree of suspicion and good clinical acumen as in this case, followed by a timely workup can differentiate the normal pregnancy changes from those suggestive of an underlying cardiac condition and hence make the difference between life and death for these mothers. In our case although the patient had minimum symptoms she was appropriately investigated and referred to a tertiary centre for further management. An intense multidisciplinary teamwork helped us in optimising the patient outcome.

\section{Funding: No funding sources \\ Conflict of interest: None declared \\ Ethical approval: Not required}

\section{REFERENCES}

1. Pieper PG, Hoendermis ES. Pregnancy in women with pulmonary hypertension. Neth Heart J. 2011;19:504-8.

2. Olsson KM, Channick R. Pregnancy in pulmonary arterial hypertension. Eur Respir Rev. 2016;25(142): 431-7.

3. Rubin LJ. Primary pulmonary hypertension. N Engl J Med. 1997;336:111-7.

4. Smith JS, Mueller J, Daniels CJ. Pulmonary arterial hypertension in the setting of pregnancy: a case series and standard treatment approach. Lung. 2012;190(2):155-60.

5. Bonnin M, Mercier FJ, Sitbon O, Roger-Christoph S, Jaïs X, Humbert $M$, et al. Severe pulmonary hypertension during pregnancy: mode of delivery and anesthetic management of 15 consecutive cases. Anesthesiology. 2005;102(6):1133-7.

6. Duarte AG, Thomas S, Safdar Z, Torres F, Pacheco LD, Feldman J, et al. Management of pulmonary arterial hypertension during pregnancy: a retrospective, multicenter experience. Chest. 2013;143(5):1330-6.

7. Roberts NV, Keast PJ. Pulmonary hypertension and pregnancy-a lethal combination. Anaesth Intensive Care. 1990;18:366-74.

Cite this article as: Banerjee R, Rahman F. A rare case of severe pulmonary hypertension in pregnancy. Int J Reprod Contracept Obstet Gynecol 2020;9:4707-9. 